\title{
Glass Welding with Ultrashort Laser Pulses Producing mm-long Filaments
}

\author{
Dirk Nodop $^{* 1}$, Paul Wiemuth ${ }^{2}$, Jan Rücker ${ }^{1}$ and Martin Kahle ${ }^{1}$, \\ ${ }^{1}$ ifw Jena - Günter-Köhler-Institut für Fügetechnik und Werkstoffprüfung GmbH, Germany \\ ${ }^{2}$ Friedrich Schiller University of Jena, Otto-Schott-Institute of Materials Research, Germany \\ *Corresponding author's e-mail: Dnodop@ifw-jena.de
}

\begin{abstract}
The welding of glass with ultrashort pulsed lasers (USP) is usually conducted with optics of high numerical aperture (NA), e.g. microscope objectives. These optics have a working distance of millimeters, limiting the thickness of the workpiece. In addition, they prevent the use of galvanometer scanners, limiting the feed rate of the process. As a step to increase the possible dimensions of the welding partners and the process speed to industrial levels, we present USP-welding with a galvoscanner and an F-theta-lens of $65 \mathrm{~mm}$ focal length. Differences between the well-known breakdown-regime and the filamentation-regime shown here regarding the growth of the molten zone and the scaling laws are observed and discussed. The substantial thickness of the samples enables classical mechanical tensile tests with high precision, the results of which are presented.
\end{abstract}

DOI: $10.2961 /$ jlmn.2020.03.2001

Keywords: USP, glass, welding, filamentation, tensile test

\section{Introduction}

Glass is a unique technical material. Its chemical resistance, dielectric strength, optical transparency and the possibility of optical imaging make it indispensable for the application of modern technologies. Especially in the chemical industry, pharmacy, optical communication technology, photonics and sensor technology, glasses are used in almost any product.

The industrial joining of glasses is a challenge even with the latest technology. If the joining zone is supposed to have the same properties as the surrounding glass, classic joining methods cannot be used. Due to the hardness of glasses, mechanical processes cannot be carried out tightly. Gluing and soldering bring foreign materials into the joining zone, which negate the favorable properties of the glass with respect to the entire workpiece. The use of glass solders is possible, but usually requires the use of different types of glass and a strong heating of the entire workpiece, which often leads to mechanical stresses and a high energy and time requirement.

Even with conventional welding of glasses by means of $\mathrm{CO}_{2}$-lasers, the problem is that either the entire workpiece has to be heated, or the area around the joining zone is subjected to strong mechanical stresses due to the thermal expansion of the glass. Some of these tensions remain in the workpiece even after the joining process. Furthermore, the partial melting of the workpieces changes their outer shape, which significantly increases the manufacturing tolerances.

In recent years, local glass welding with ultra-short pulse lasers (USP) has been discussed in scientific publications $[1,2,3]$. This process is based on a spatially very limited energy input, which creates molten zones with a diameter of approximately $100 \mu \mathrm{m}$. By lining up these welding zones, flat connections can be made. Since the process works through the transparent glass, interfaces can be welded that are not mechanically accessible. Due to the very small heat affected zone, glasses with very different expansion coefficients and assemblies with temperaturesensitive elements can also be joined. One of the biggest limitations of this joining technology is that the interfaces of the joining partners have to be in optical contact beforehand, which placed high demands on the surface quality of the joining zone. As shown later by several groups, the USP-welding process is able to bridge and even reduce gaps of several micrometers between the interfaces $[2,4]$.

A current overview of the field can be found in [5]. In this publication, several current studies on gap-bridging welding are presented, the formation of cracks is discussed and simulations on thermal effects are presented. Accordingly, tensile stresses and cracks in the weld seam arise especially when bridging gaps. These can be reduced by increasing the temperature - and thus the viscosity - of the melting zone only to such an extent that the viscous material bridges the gap directly vertically, but not to the extent that it fills it sideways.

Another hurdle to the industrial usage of the process is its productivity. Fixed, high-numerical-aperture (high-NA) optics were used in the literature, and the glasses were moved with linear axes, which greatly limits the possible feed rates and usable laser powers. The high-NA optics and low laser pulse energies of up to several microjoules are used to enable optical breakdown in the glass while circumventing self-focusing [5].

The classical UPS-welding process described in the literature is based on optical breakdown. Therefore, the laser radiation is focused into the transparent material. At optical intensities in the order of $10^{13} \mathrm{~W} / \mathrm{cm}^{2}$ (depending on wavelength and material) multiphoton absorption occurs and generates free electrons. These electrons can then directly absorb more energy from the light field. For long pulses, avalanche-ionization can also play an important role [6]. On a timescale of several picoseconds, the electrons trans- 
fer their heat to the glass lattice. At high enough repetition rates, subsequent pulses can heat the glass lattice to the melting point while the low thermal conductivity limits the molten zone well below millimeter-size.

For the initial focusing, high-NA objectives are used to prevent the ablation of the upper glass surface by lowering the optical intensity there through the high convergence. In addition, the high NA allows to reach the intensity threshold for optical breakdown while staying below the power threshold for self-focusing.

The welding under self-focusing conditions has also been investigated: As described in [7], it has been used for reinforcing direct bonds, increasing their shear strength by a factor of three.

In [8] the authors describe experiments on long-focal USP glass welding. Welding speeds of up to $1 \mathrm{~m} / \mathrm{s}$ could be achieved. They report that their weld seams in fused silica reach strengths of up to $30 \mathrm{MPa}(60 \%$ of fused silica), but without presenting the measurement setup in detail or making a connection to the laser parameters. In addition, the authors propose that residual stresses seem to be reduced under certain parameters since the heating rate is reduced for bigger foci.

The authors of [9] report on the welding with Bessel beams, leading to elongated molten zones up to $0,5 \mathrm{~mm}$, relaxing the positioning requirements. The applied welding speed was $0,2 \mathrm{~mm} / \mathrm{s}$.

In [10], the authors compare optical breakdown with filamentation, which is the interplay between self-focusing and diffraction (natural diffraction or diffraction in breakdown-generated plasmas), leading to very elongated foci: For 45-fs-pulses for example, the threshold energy for filamentation is lower than for pure breakdown when focusing lengths above approximately $45 \mathrm{~mm}$ are used. That means that the beam is influenced by self-focusing, reducing the size of the initial focus. The generated plasma acts as a diverging lens which increases the beam size. When leaving the plasma, the beam will self-focus again, defocus again, etc., until the energy loss by the plasma generation reduces the pulse energy below either threshold. Of course, this "breaking point" of $45 \mathrm{~mm}$ focal length reduces with increased pulse length since the breakdown-threshold is intensity-dependent while the threshold for self-focusing (not the magnitude) is only dependent on the optical power [11].

Therefore, the goal of this work is the transition to welding with long focal lengths to enable the use of galvoscanners and thicker work pieces. This further relaxes the positioning requirements due to the long molten zones and enables very high welding speeds up to $1 \mathrm{~m} / \mathrm{s}$. Another more indirect aim is to increase the usable average laser power, since industrial USP-Lasers are available up to hundreds of watts, where the power/price-ratio increases with the average power. The higher process speeds enabled by galvoscanners are intended to increase the economics of the process to such an extent that mass production, for example in microfluidics or optics production, container construction, fiber technology, micro technology and sensor technology, becomes realistic.

\section{Experimental setup}

The following experiments were conducted with an "Amphos 100 flex" laser system with pulse energies up to $10 \mu \mathrm{J}$, a repetition rate of $1 \mathrm{MHz}$, a wavelength of $515 \mathrm{~nm}$ and a pulse duration of $900 \mathrm{fs}$. The laser beam was directed through a scanlab intelliscan 14 galvoscanner. An F-thetalens of $65 \mathrm{~mm}$ focal length focused the beam down to a measured spot size of $17 \mu \mathrm{m}$ including the enlargement by the refractive index of the workpiece but not including the effect of the Kerr lens.

For the first experiments, UV-grade fused silica was chosen as the workpiece material. Mirror substrates of 1inch diameter and $6 \mathrm{~mm}$ thickness with a surface flatness of $\lambda / 8$ and 20/40 S-D were used. They were cleaned with isopropanol, blown dry, placed and gently pressured on each other by hand for optical contacting. No heat was applied. Since the contact area had to be reduced for the tensile tests, the surface of one of the samples was roughened by a laser treatment beforehand, so that only a circle of $16 \mathrm{~mm}$ diameter got into optical contact with the other sample (see Fig. 1)

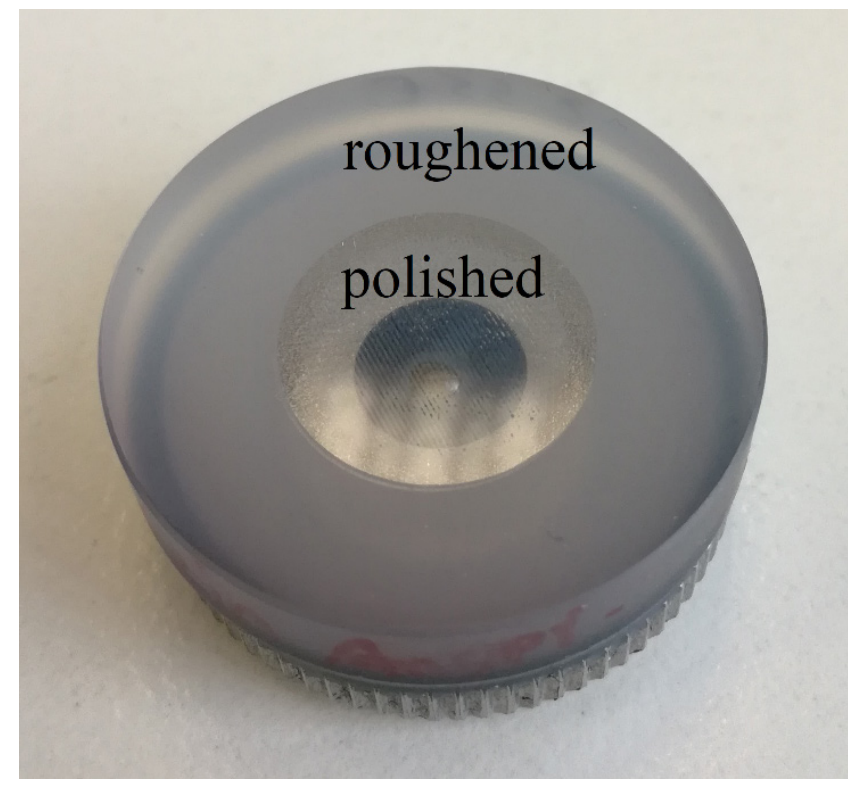

Fig. 1 Fused silica sample with reduced optical contacting zone.

Then, the entire contact area was welded with a distance of $200 \mu \mathrm{m}$ between the welding lines in order to prevent mutual influence of the welding seams (see Fig. 2). After this processing, the samples were examined in different ways: Some of the samples were cut for optical microscopy of the welding seams. For this purpose, the edges were submerged in glycerol for index matching in order to avoid polishing. The rest of the samples was glued with DELO AD840 adhesive to M6 stainless steel DIN 467 knurled nuts (see Fig. 3) for testing on a tensile testing machine (Instrom 4411). Both knurled nuts were bolt with M6 threaded bars with a length of $100 \mathrm{~mm}$, and roughly the last $10 \mathrm{~mm}$ of the bars were clamped into the tensile testing machine. With this measure, it was ensured that the flexibility of the bars would largely compensate for any shear forces caused by a lack of centricity of the glued items. Since the glue had a lower strength than the weld, the 
welded area had to be reduced as described above. In this way, the weld will always break before the glue.

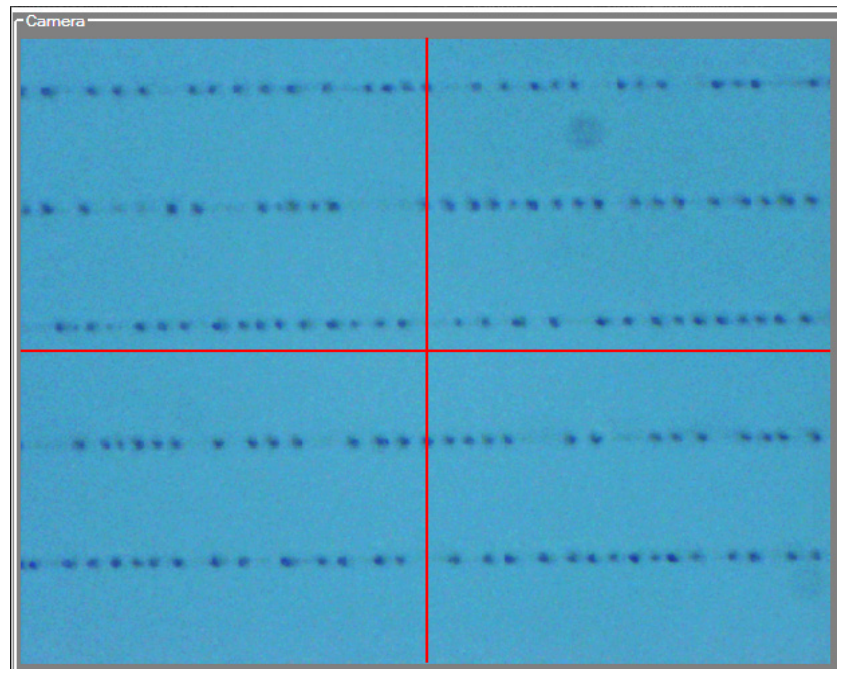

Fig. 2 Top view of the welding seams. Every black spot is a small bubble, which is the beginning of a filament. laser parameters were $\mathrm{f}_{\mathrm{P}}=1 \mathrm{MHz}, \mathrm{P}=5 \mathrm{~W}, \mathrm{t}_{\mathrm{P}}=0,9 \mathrm{ps}$.

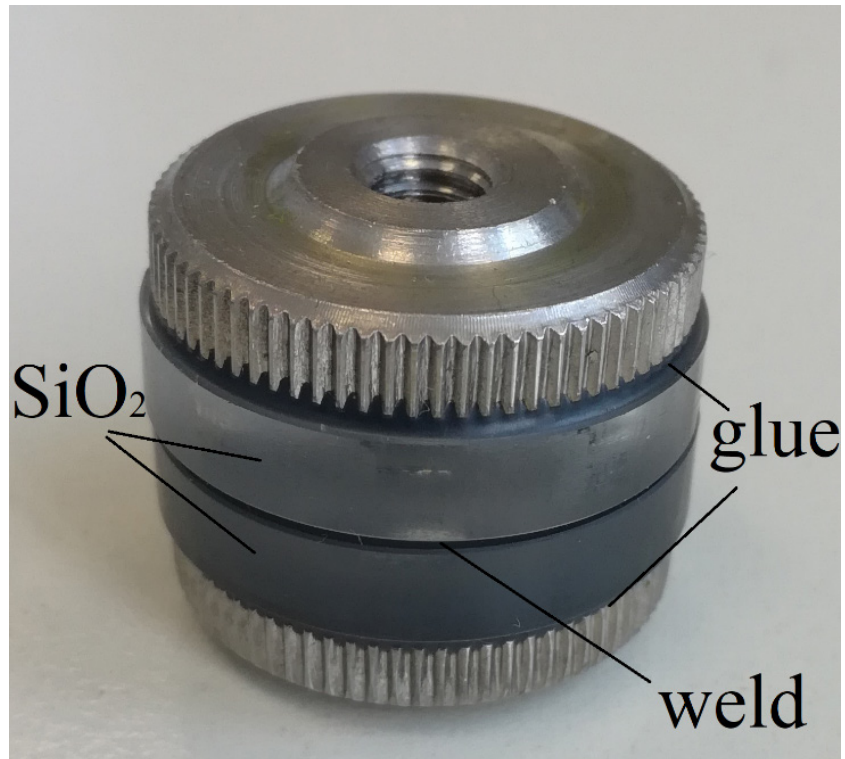

Fig. 3 Welded fused silica substrates glued to knurled nuts for tensile testing.

\section{Results}

As a first step, the laser focus was placed in the volume of the upper sample rather than close to the interface of the samples to examine the molten zones. Fig. 4 shows a microscopic sideview of one of the lines seen in Fig 2. Here, the laser entered the sample from the bottom, creating a modification. Due to the measurement setup the modification could only be detected be stray light. Towards the laser irradiation the modifications show a small bubble (visible as a white spot). After this bubble the samples show a filament-like modification. The stray light hints to an index modification and smaller bubbles in the filament. The maximum length of such an entity in this sample is $>800 \mu \mathrm{m}$, even though one can see that not all bubbles occur at the same height. It is possible, that the actual modification is longer than measured here, but not visible under optical microscopy.

The bubbles seem to be characteristic for fused silica. These bubbles could not be observed for other investigated materials like BK7 or Zerodur. While the formation of the bubbles seems like a drawback for the application of the welding technique, they make it much easier to find the laser treated area in the analysis. As other authors have pointed out, the bubbles probably result from small amounts of oxygen in the fused silica material [12]. This oxygen is set free in gaseous form during the melting and solidification. The lines below the bubbles are modifications of the refractive index. The material itself is transparent. The bright appearance of the modifications is a consequence of their focusing capabilities due to the index modification. As the authors of [12] point out, the oxygen bubbles are possibly able to move in the melt, so that the bubbles seen in Fig. 2 might be the collection of all the oxygen that was produced in the vertical modification. During the welding, the laser beam hit the sample from above so that the oxygen would travel towards the laser beam.

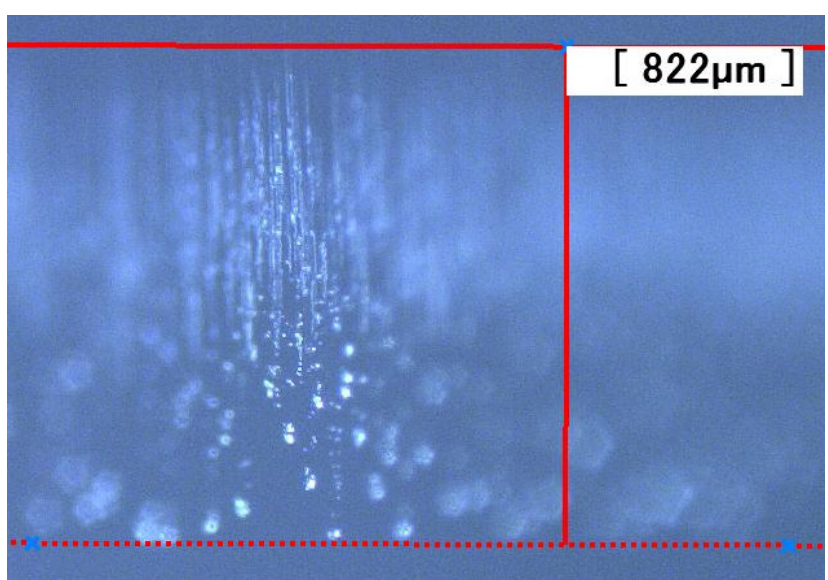

Fig. 4 Microscopic sideview of laser modifications in fused silica: $\mathrm{fP}_{\mathrm{P}}=1 \mathrm{MHz}, \mathrm{EP}=5 \mu \mathrm{J}, \mathrm{v}_{\mathrm{scan}}=1 \mathrm{~mm} / \mathrm{s}$.

Under the same conditions, a higher pulse energy can lead to irregular behavior of the filaments, as can be seen in Fig. 5.

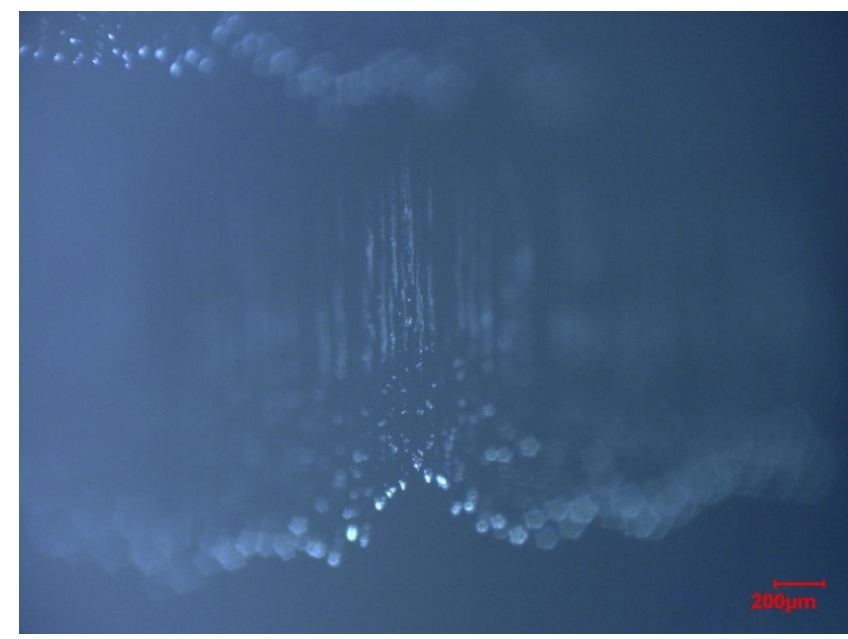

Fig. 5 Microscopic sideview of laser modifications in fused silica: $\mathrm{fP}_{\mathrm{P}}=1 \mathrm{MHz}, \mathrm{Ep}=10 \mu \mathrm{J}, \mathrm{v}_{\mathrm{scan}}=1 \mathrm{~mm} / \mathrm{s}$. 
Here, an irregular behavior of the filament length and the starting depth of the filament is visible. To further investigate this observation, the filament length and the depth of the filament were evaluated in dependence of the pulse energy.

\subsection{Filament length vs. pulse energy}

At first, the pulse energy was varied over multiple samples under constant geometrical conditions. Fig. 6 shows the results regarding the length: Our first observation was the clear inset of self-focusing at about $1 \mu \mathrm{J}$ independent of the feed-rate $v_{\text {scan }}$. This is in accordance with self-focusing threshold calculated via Equation (1) [11]:

$$
P_{\text {crit }}=\frac{0,148 \lambda^{2}}{n n_{2}}
$$

With the laser wavelength $\lambda=515 \mathrm{~nm}$ used here, the refractive index of fused silica $n=1,4615$ as well as the nonlinear index $\mathrm{n}_{2}=2,44 \cdot 10^{-20} \mathrm{~m}^{2} / \mathrm{W}[13]$ and the pulse length of $900 \mathrm{fs}$ used here this results in an energy threshold of about $1 \mu \mathrm{J}$. Below this energy, no self-focusing occurs. Without self-focusing the intensity is too low for nonlinear absorption and the laser light is transmitted.

When comparing this with the results of Nguyen [10], we deduct that at this point, we are in the regime of pure filamentation. Higher pulse energies allow for longer filaments. The physical reason for this is that both the self-focusing and the nonlinear-absorption are dependent on the pulse energy, former in a linear way and latter in a quadratic way [11]. Since the laser pulses loose energy along the axis through the generation of the plasma channel, after a certain length, the pulse energy is too low for self-focusing. Interestingly, this dependence seems to be in good approximation linear up to a certain scanning speed, as depicted in Fig. 6.

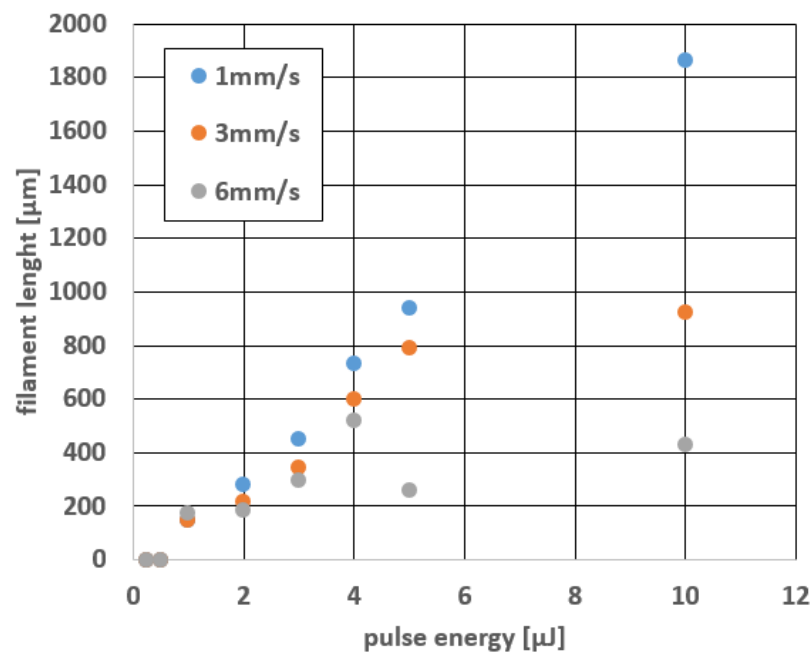

Fig. 6 Dependence of the filament length on the pulse energy for different feed-rates at $1 \mathrm{MHz}$ repetition rate.

Fig. 6 also shows that the filaments do not reach their full possible length when the scanning speed is too high. We propose the following reason for this behavior: While the beam path and the plasma channel are similar for the first laser pulse and later pulses, the upper part of the plasma channel absorbs more energy, since the pulse energy decreases along the beam path. Because of this, the glasslattice in the upper part of the plasma channel heats up more rapidly than in the lower part. When the feed-rate is too high, too little pulses are irradiating this one channel, so that the heat accumulation in the lower part of the filament is not sufficient to reach the melting temperature. The temperature difference along the filament might have an impact on the optical path of the pulses, but we suspect that this influence is small compared to the self-focusing and plasma-diffraction forming the filament.

From the perspective of energy, one could simply say that the energy input over time per unit length has to be high enough to melt the entire plasma channel volume and not just the upper part of it.

\subsection{Filament position vs. pulse energy}

With the same samples, the position of the filament was measured with the sample surface as a reference. The position of the geometrical focus to the sample surface was constant in the experiments. As can be seen in Fig. 7, the feed-rate has no influence on the position. It is obvious, that the position of the filament changes linear with the pulse energy in the direction of the laser source. Although this is known from USP-welding with microscope objectives, the magnitude of the effect is completely different here. With high-NA-optics, the molten zone grows with the pulse energy towards the laser source in the order of $100 \mu \mathrm{m}$. Here, the position changes in the direction of the laser source over several millimeters. The reason for this behavior is the self-focusing. The linear dependence is in accordance with the theory, since the dioptric power of a Kerr lens is proportional to the optical power of the beam [11].

The remarkable result here is that the filament position is easily controllable despite the Kerr lensing and independent of the scanning speed, which -together with the long working distance- provides easy alignment in the application.

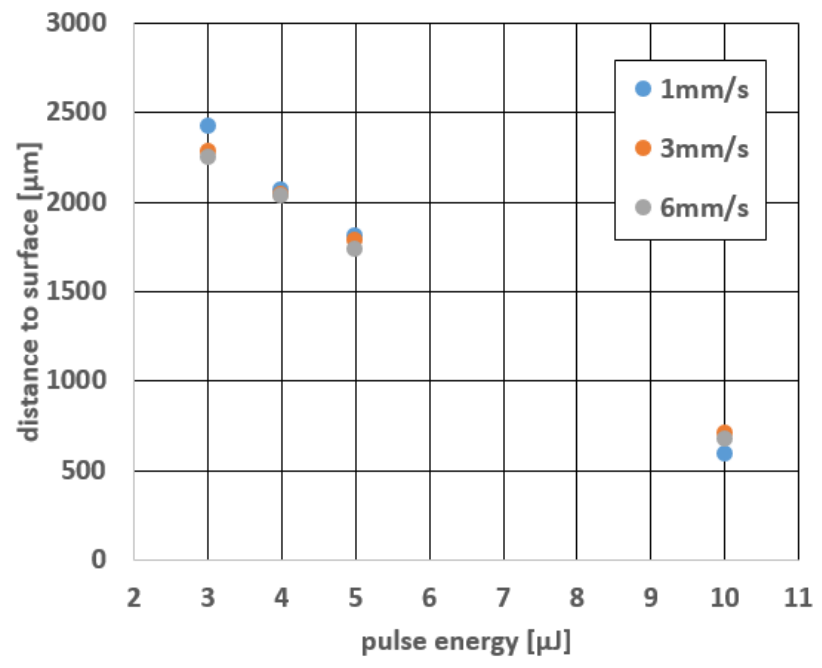

Fig. 7 Dependence of the filament position on the pulse energy for different feed-rates at $1 \mathrm{MHz}$ repetition rate. 


\subsection{Strength over the length of the filament}

When one thinks about the application of the comparably long filament for welding, the next question after the alignment is the usability of the filament length for the strength of the bond. Because of that, we produced welds with constant parameters $(5 \mu \mathrm{J}, 1 \mathrm{MHz}, 1 \mathrm{~mm} / \mathrm{s})$ but different positions of the filament with respect to the sample interface. These samples were tested for their tensile strength as described above. The results can be seen in Fig. 8.: The samples welded with the upper part of the filament show a breaking strength of over $2 \mathrm{kN}$ which means a pressure of $10 \mathrm{~N} / \mathrm{mm}^{2}$ respective to the entire welded area. This is about $20 \%$ of the strength of the volume material and 20 times more than the strength of the optically contacted samples without the weld.

As shown in Fig. 2 the geometry of the welding seams is far from optimized, so there is substantial room for improvement here. The important thing that this measurement shows however is the fact that this strength decreases only slowly towards the lower part of the filament. Several hundred micrometers away from the optimal welding position of the filament, the strength is still about $50 \%$ of the maximum value. This shows that the majority of the filament length is actually usable for welding, which strongly relaxes the required precision of the alignment and enables welding with galvoscanners.

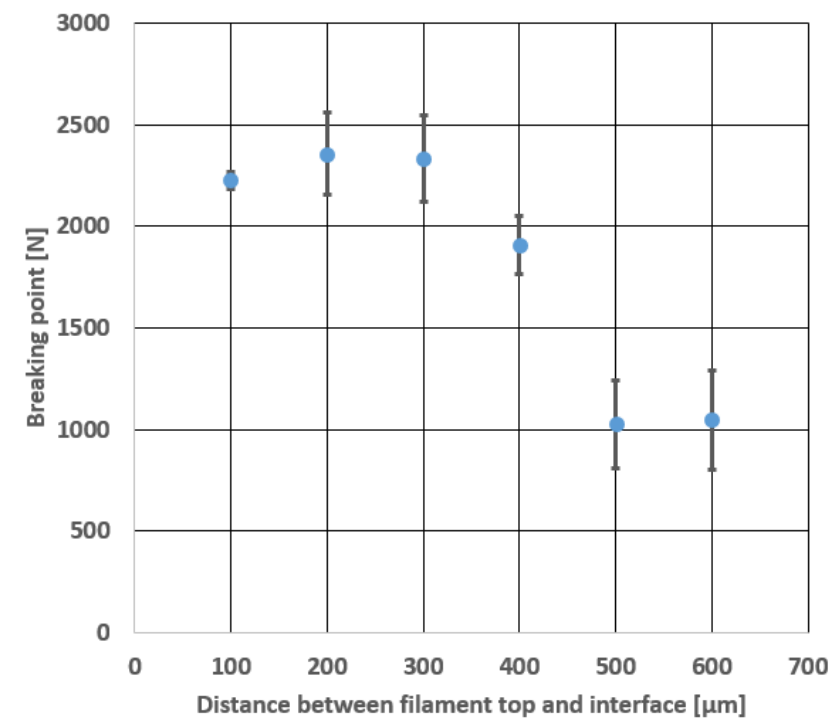

Fig. 8 Dependence of the breaking force on the position of the filament with respect to the interface.

As a side note, some of the welds produced were tested for their He-leakage according to a DIN EN 1779 leakage test (ASM 310 Pfeiffer Vacuum). None of the samples transmitted a measurable amount of helium, which is not surprising since the samples were in optical contact before welding.

\section{Conclusion and Outlook}

USP-welding in the filamentation regime was investigated and demonstrated. We have shown that the process of welding with filaments starts when the pulse energy respectively the peak power exceeds the critical power known for self-focusing. Above that energy the length of the modification increases linearly with the pulse energy as long as the feed rate is low enough for the heat accumulation to melt the whole length of the filament.

With long focal lengths comparably long molten zones of $2 \mathrm{~mm}$ can be generated. Furthermore, the position of these molten zones is stable and controllable despite the fact that the process is dominated by Kerr lensing. Finally, it was shown that the bonding strength of the weld changes continuously when different sections of the filaments are used for welding the interface. $20 \%$ of the breaking strength of the fused silica base material were reached with an un-optimized weld geometry. This tolerant behavior, together with the long working distances (here $65 \mathrm{~mm}$ ) ensures easy alignment and paves the way for industrial scale applications of ultrafast laser glass welding with galvoscanners.

Future work will include a more detailed analysis of the modifications to separate between the bubbles and the in dex change, also measuring the width of the modification. Further experiments will target on the maximum length of the filaments. Early parameter scans have shown, that even longer molten zones up to $8 \mathrm{~mm}$ are possible. The limits of this process have to be found and explained. Furthermore, the process instabilities shown in Fig. 5 will be investigated and of course the bonding strength and process speed will be optimized.

\section{Acknowledgments}

This work was supported by the German Federal Ministry for Economic Affairs and Energy under the identifier 49VF170036.

\section{References}

[1] T. Tamaki, W. Watanabe, J. Nishii, and K. Itoh: Jpn. J. Appl. Phys., 44, (2005) L687.

[2] K. Cvecek, R. Odato, S. Dehmel, I. Miyamoto, and M. Schmidt: Opt. Express, 23, (2015). 5681.

[3] S. Richter, F. Zimmermann, A. Tünnermann, and S Nolte: Opt. Laser Technol., 83, (2016), 59.

[4] S. Richter, F. Zimmermann, R. Eberhardt, A. Tünnermann, and S. Nolte: Appl. Phys. A, 121, (2015), 1.

[5] K. Cvecek, S. Dehmel, I. Miyamoto, and M. Schmidt: Intern. J. Extreme Manuf., 1, (2019), 042001.

[6] J. Noack, and A. Vogel: IEEE J. Quantum Electron., 35, (1999), 1156.

[7] D. Hélie, F. Lacroix, and R. Vallée: J. Laser Micro/Nanoengin., 7, (2012), 284.

[8] M. Gstalter, G. Chabrol, A. Bahouka, K.D. Dorkenoo, J.L. Rehspringer, and S. Lecler: Appl. Opt., 58, (2019). 8858.

[9] G. Zhang, R. Stoian, W. Zhao, and G. Cheng: Opt. Express, 26, (2018), 917.

[10]N.T. Nguyen, A. Saliminia, S.L. Chin, and R. Vallee: Proc. of SPIE, Vol. 5578, (2004). 665.

[11]R. Paschotta: "Encyclopedia of laser physics and technology (Vol. 1). (Wiley-vch, Berlin, 2008).

[12]K. Cvecek, I. Miyamoto, and M. Schmidt: Opt. Express, 22, (2014), 15877.

[13] H. Garcia, A.M. Johnson, F.A. Oguama, S. Trivedi: Opt. Lett., 28, (2003). 1796.

(Received: June 26, 2020, Accepted: September 24, 2020) 